\title{
Leveraging shared caches for parallel temporal blocking of stencil codes on multicore processors and clusters
}

\author{
Markus Wittmann, Georg Hager, Jan Treibig, Gerhard Wellein \\ Erlangen Regional Computing Center \\ University of Erlangen-Nuremberg \\ Martensstr. 1, 91058 Erlangen, Germany
}

June 15, 2010

\begin{abstract}
Bandwidth-starved multicore chips have become ubiquitous. It is well known that the performance of stencil codes can be improved by temporal blocking, lessening the pressure on the memory interface. We introduce a new pipelined approach that makes explicit use of shared caches in multicore environments and minimizes synchronization and boundary overhead. Benchmark results are presented for three current x86-based microprocessors, showing clearly that our optimization works best on designs with high-speed shared caches and low memory bandwidth per core. We furthermore demonstrate that simple bandwidth-based performance models are inaccurate for this kind of algorithm and employ a more elaborate, synthetic modeling procedure. Finally we show that temporal blocking can be employed successfully in a hybrid shared/distributed-memory environment, albeit with limited benefit at strong scaling.
\end{abstract}

\section{Introduction}

\subsection{The Jacobi stencil}

Stencil computations are central to many scientific and technical applications, especially when solving partial differential equations on regular lattices. Even large-scale multigrid solvers employ smoothing steps comprising stencil updates following the Gauss-Seidel or Jacobi schemes [1]. Standard optimization techniques like spatial blocking are usually applied to ensure optimal spatial locality of data accesses. The advent of multicore chips and advanced architectures like GPUs and the Cell processor has recently initiated a new interest in stencil optimizations [2, 3].

The Jacobi algorithm is a simple method for solving boundary value problems. Although it is not too useful as a numerical algorithm by itself, it is the central component of many multigrid solvers. Moreover it can serve as a prototype for more advanced stencil-based methods like the lattice-Boltzmann algorithm (LBM). In three dimensions, one stencil ("cell") update is described by

$$
B_{i, j, k}=\frac{1}{6}\left(A_{i-1, j, k}+A_{i+1, j, k}+A_{i, j-1, k}+A_{i, j+1, k}+A_{i, j, k-1}+A_{i, j, k+1}\right) .
$$




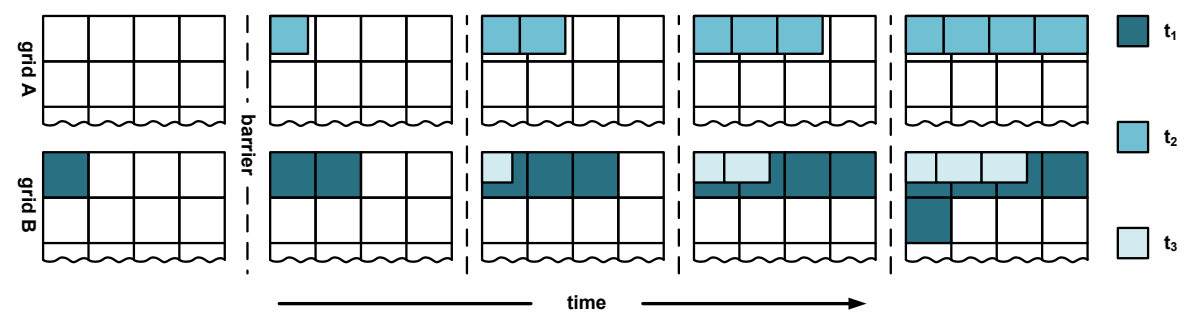

Figure 1: Temporal blocking by pipelining, shown here for three threads in two dimensions and separate grids $A$ and $B$. All threads in a team update the most part of a block consecutively. Shifting the block by one cell in each direction after an update avoids extra boundary copies. To avert race conditions, a global barrier is required after each block update. This scheme can be easily generalized to support multiple updates per thread. Possible optimizations include the use of a "compressed grid" update scheme, and relaxed synchronization (see text).

Successive sweeps over the computational domain are performed to reach convergence, writing to grids $A$ and $B$ in turn. Counting the usually required cache line allocate on a write miss, the kernel has a naive code balance of $B_{\mathrm{c}}=8 / 6 \mathrm{~W} / \mathrm{F}$ (double precision words per flop). However, the actual number of words transferred over the slowest data path (the memory bus) can be reduced to three per stencil update with a suitable spatial blocking scheme. Furthermore, the write allocate can be circumvented on current x86-based processors by employing non-temporal store instructions, which bypass the cache hierarchy. These and other well-known standard optimizations like data alignment and SIMD vectorization have been applied for our baseline version of the algorithm and are described in the literature [2, 3, 4]. We use OpenMP-parallel C/C++ code whenever possible, and revert to compiler intrinsics or assembly language only if necessary, e.g., if the compiler refuses SIMD vectorization because a loop runs backward (as is the case with the compressed grid version of pipelined temporal blocking, which will be described in Sect.2).

\subsection{Baseline and test bed}

The memory-bound performance of the baseline code on a given architecture can be easily estimated by assuming that memory bandwidth is the sole limiting factor, and that all other contributions can be hidden behind it. This assumption is valid for current multicore processors if all cores sharing a memory interface are used in a parallel calculation, but may be false for single-threaded code [12]. See Sect. 2.3 for a discussion of this problem.

If the achievable STREAM COPY bandwidth (using non-temporal stores) is $M_{\mathrm{s}}$, a "perfect" baseline Jacobi code $\left(B_{\mathrm{c}}=0.33 \mathrm{~W} / \mathrm{F}\right)$ should show a performance of

$$
P_{0}=\frac{M_{\mathrm{s}}}{16 \text { bytes }}[\mathrm{LUP} / \mathrm{s}] \text {. }
$$

We use the "lattice site updates per second" (LUP/s) metric here. Benchmark tests were performed on a variety of systems, which are briefly described in Table 1 together with measurements for different low-level benchmarks, first and foremost the STREAM 
Table 1: Overview on cache group structure, STREAM COPY (array size of 20,000,000 elements), and update performance for the systems in the test bed. Nontemporal stores were used for the STREAM COPY, so all bandwidth numbers denote actual bus traffic.

\begin{tabular}{|c|c|c|c|}
\hline Name & $\begin{array}{l}\text { Nehalem } \\
\text { EP }\end{array}$ & $\begin{array}{l}\text { Nehalem } \\
\text { EX }\end{array}$ & Istanbul \\
\hline \multirow{3}{*}{ Type } & Xeon & Xeon & Opteron \\
\hline & X5550 & X7560 & 2435 \\
\hline & @ $2.66 \mathrm{GHz}$ & @ $2.27 \mathrm{GHz}$ & @ $2.6 \mathrm{GHz}$ \\
\hline Cores & 4 & 8 & 6 \\
\hline L1 size $[\mathrm{kB}]$ & 32 & 32 & 64 \\
\hline L2 size $[\mathrm{kB}]$ & 256 & 256 & 512 \\
\hline L3 size $[\mathrm{MB}]$ & 8 & 24 & 5 \\
\hline L3 cache group [cores] & 4 & 8 & 6 \\
\hline Sockets & 2 & 4 & 2 \\
\hline$M_{\mathrm{s}}[\mathrm{GB} / \mathrm{s}]$ & 19.0 & 7.9 & 10.5 \\
\hline$M_{\mathrm{um}, 1}[\mathrm{~GB} / \mathrm{s}]$ & 16.2 & 7.0 & 6.9 \\
\hline$M_{\mathrm{uc}, 1}[\mathrm{~GB} / \mathrm{s}]$ & 28.3 & 25.0 & 15.7 \\
\hline$M_{\mathrm{uc}, \max }[\mathrm{GB} / \mathrm{s}]$ & 51.2 & 176.2 & 74.8 \\
\hline
\end{tabular}

COPY, $A(:)=B(:)$ The remaining benchmark numbers were obtained using a special array update loop, which is described in Sect. 2.3

All systems are of ccNUMA type, so the usual precautions regarding page placement apply. The Nehalem EX node is an early access (EA) system provided by Intel, with half the memory boards removed. Consequently, it has only half the main memory bandwidth of a fully equipped system. It is worth noting that Nehalem EX features a redesigned L3 cache with a "banked" structure, which leads to a very high cache bandwidth. The consequences of this peculiarity will be investigated in Sections 2.3 and 2.4 .

For benchmarking the distributed-memory algorithm described in Sect. 3 we use a Nehalem EP cluster (node parameters as above) with a fully nonblocking fat-tree QDR-InfiniBand network.

The idea behind temporal blocking is to perform multiple in-cache updates on each grid cell before the result is evicted to memory, thereby reducing effective code balance. Section 2 will introduce a pipelined temporal blocking scheme in a shared-memory parallel context, while Sect. 3 describes how and under what conditions these optimizations can be put to use in a hybrid (shared/distributed-memory) code.

\subsection{Related work}

Improving the performance of stencil codes by temporal blocking is not a new idea, and many publications have studied different methods in depth [6, 7, 8, 9, 10]. Conventional temporal blocking performs multiple updates on a small block of the computational domain before proceeding to the next block [8]. This strategy has the important drawback that in-cache stencil updates are not naturally overlapped with loads and stores to main memory, leading to under-utilization of the memory interface. Cache-oblivious algorithms as proposed by Frigo et al. [9] have the attractive property of being independent 
of cache sizes, but they come at the cost of irregular block access patterns, which cause many data TLB misses. This was shown for a 3D lattice Boltzmann (LB) application kernel in Ref. [11].

However, the explicit use of shared caches provided by modern multicore CPUs has not yet been investigated to great detail. Ref. [4] describes a "wavefront" method similar to the one introduced here. However, that work was motivated mainly by the architectural peculiarities of multi-core CPUs, and does not elaborate on specific optimizations like avoiding boundary copies and optimizing thread synchronization. Our investigation is more general and explores a much larger parameter space. Finally there is, to our knowledge, as yet no systematic analysis of the prospects of temporal blocking on hybrid architectures, notably clusters of shared-memory compute nodes.

\section{Shared-memory pipelined temporal blocking on shared caches}

\subsection{Algorithm and in-cache optimizations}

In contrast to previous approaches to cache reuse with stencil algorithms, pipelined temporal blocking makes explicit use of the cache topology on modern processors, where certain cache levels are shared by groups of cores, which we call cache groups. On all test systems used here (see Sect.[1.2), the outer cache level (L3) is shared by all cores on a socket.

Pipelined blocking splits the set of all available threads into teams of size $t$, where a team runs on cores sharing a cache. It is possible that the size of a team is smaller than the whole cache group, but this option will not be explored here. Each team has one "front" thread, which performs the first $T$ updates on a certain grid block (see Fig. 1 for a visualization with three threads and $T=1$ ). Of course the block is loaded to cache in this process, and if it is small enough, the remaining threads can perform further updates ( $T$ each) on it in turn before it gets evicted to memory. All threads in the team can be kept busy by keeping this pipeline running, with different blocks in different stages until each block of the whole computational domain has been updated $t \cdot T$ times, which completes a team sweep. To avoid race conditions, the minimum distance between neighboring threads is one block, but it may be larger. An estimate for the maximum distance is given by the cache size divided by $t$ times the size of one block. Due to the one-layer shift after each block update, the actual amount of cache needed is actually larger, depending on the blocksize and the overall number of updates, $t \cdot T$. In the simplest case, the distance is kept constant by imposing a global barrier across all threads after each block update. See below for ways to reduce synchronization overhead.

Pipelined blocking can potentially overlap data transfer and calculation, because the front thread continuously operates on new blocks, which have to be fetched from memory. Compared to the wavefront technique [4], it does not incur extra work or boundary copies. In order to achieve optimum in-cache performance, the kernel must be fully SIMD-vectorized, i.e., packed arithmetic (addpd/mulpd) and packed loads and stores are essential. Although the performance penalty for unaligned moves from or to L1 cache is small on the most current $x 86$ architectures, we have also made sure that all loads and stores are aligned to 16-byte address boundaries, and the corresponding instructions are of the "aligned" type (movapd). 
The standard Jacobi algorithm iterates between two versions of the computational grid, one holding the current state while the other is used to store the next time step (i.e., the updated values). If the temporally blocked algorithm performs an even number of time steps on each block, the second grid becomes obsolete and can be reduced to a collection of $t-1$ small temporary arrays, which must only be large enough to hold all intermediate blocks handed down the pipeline [4]. Although this optimization reduces the required data traffic from and to main memory by a factor of two, it does not significantly cut down on in-cache transfers. However, the well-known "compressed grid" optimization can be applied here: During the first team sweep, each result is written to a location shifted by the vector $(-1,-1,-1)$ relative to its original position. In order to avoid complex address calculations, alternate team sweeps shift by $(-1,-$ $1,-1)$ and $(+1,+1,+1)$, respectively, requiring reverse loops (running from large to small indices) on all even sweeps. Since the compiler refuses to properly SIMD-vectorize the inner loop in this case, SSE intrinsics were used to get optimal code. The use of nontemporal stores is unnecessary and even counterproductive; after the $t \cdot T$ updates in a team sweep, a block gets evicted to memory automatically by the usual replacement mechanisms. The benefit of using "compressed grid" is that the second grid can be dropped altogether (even including the small temporary arrays), saving nearly half the memory. It also reduces in-cache traffic if at least two successive layers of a block fit into the $\mathrm{L} 1$ cache.

In typical shared-memory systems there is usually more than one outer-level cache group, the simplest case being a multi-socket node. Hence, more than one team must be kept running. We choose to use those additional threads to perform further updates on the blocks already handled by the "front" team. This enlarges the whole update pipeline to $n \cdot t \cdot T$ stages, where $n$ is the number of teams. Since different teams do not share any cache, blocks updated by one team must be transferred to another cache when the next team takes over. As will be shown in the results section, it makes sense to enforce a larger distance between successive teams than between neighboring threads inside a team. We call this extra distance the team delay, $d_{\mathrm{t}}$.

A problem with our method of consecutive thread teams is that every thread updates every block, rendering explicit (i.e., first touch) ccNUMA placement optimizations mostly useless. However, since the pressure on the memory interfaces is greatly reduced by the temporal blocking, a round-robin page placement strategy is adequate to achieve parallelism in memory access. Additionally, the distributed-memory parallelization described in Sect. 3 can be used to run one MPI process per socket, which alleviates the placement problem. The baseline Jacobi code does employ standard firsttouch page placement.

\subsection{Relaxed synchronization}

It is well known that synchronizing a large group of threads running on different cores can incur significant overhead. Depending on the system topology (shared caches, sockets, ccNUMA locality domains) and on the implementation used by the OpenMP compiler, a barrier may cost hundreds if not thousands of cycles [12]. With the number of cores per shared-memory node increasing steadily over time, alternatives should be used where appropriate. In our pipelined temporal blocking scheme, the barrier can be removed completely if the minimum and maximum thread distance and team delays are still observed. To this end, each thread $t_{i}$ maintains a counter variable $c_{i}$, which carries the volatile attribute and is initialized to zero at the start of each team sweep. It gets incremented whenever $t_{i}$ has updated its current block. Each $c_{i}$ is located in a 


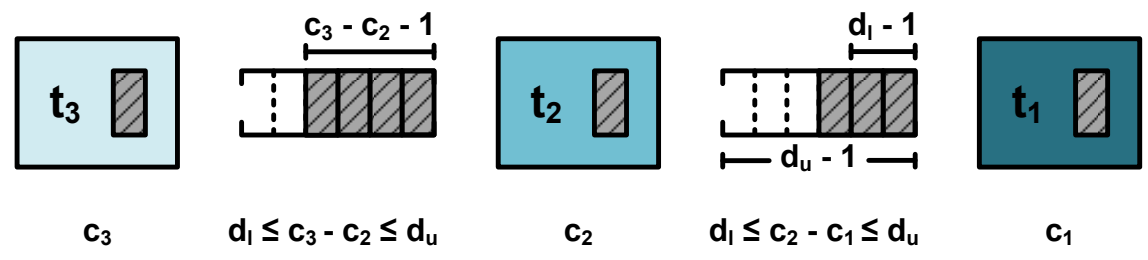

Figure 2: Relaxed thread synchronization. A global barrier is avoided by observing "soft" lower and upper limits for the distance between consecutive threads.

cache line of its own to prevent false sharing. The conditions to allow thread $t_{i}$ to start updating the next block are then

$$
c_{i-1}-c_{i} \geq d_{1} \wedge c_{i}-c_{i+1} \leq d_{\mathrm{u}} .
$$

The first condition averts data races, whereas the second maintains a maximum distance between consecutive threads. The team delay is trivially implemented by adding $d_{\mathrm{t}}$ to $d_{1}$ on a team's front thread and to $d_{\mathrm{u}}$ on its "rear" thread. Overall front and rear threads (i.e., the front thread of the first and the rear thread of the last team) ignore the first and the second condition, respectively.

In this scheme, only thread $t_{i}$ updates its own counter $c_{i}$; all others read its updated value by means of the standard cache coherence mechanisms. Making the variables volatile prevents register optimizations. The naive choice of $d_{\mathrm{l}}=d_{\mathrm{u}}=1$ imposes a rigid lock-step between threads. As will be shown in the results section, it is better to choose a different value at least for $d_{\mathrm{u}}$, allowing for some "looseness" in the pipeline.

In pseudo-code the complete algorithm for one team sweep, including the compressed grid and relaxed synchronization optimizations, looks as follows (for a team delay of zero):

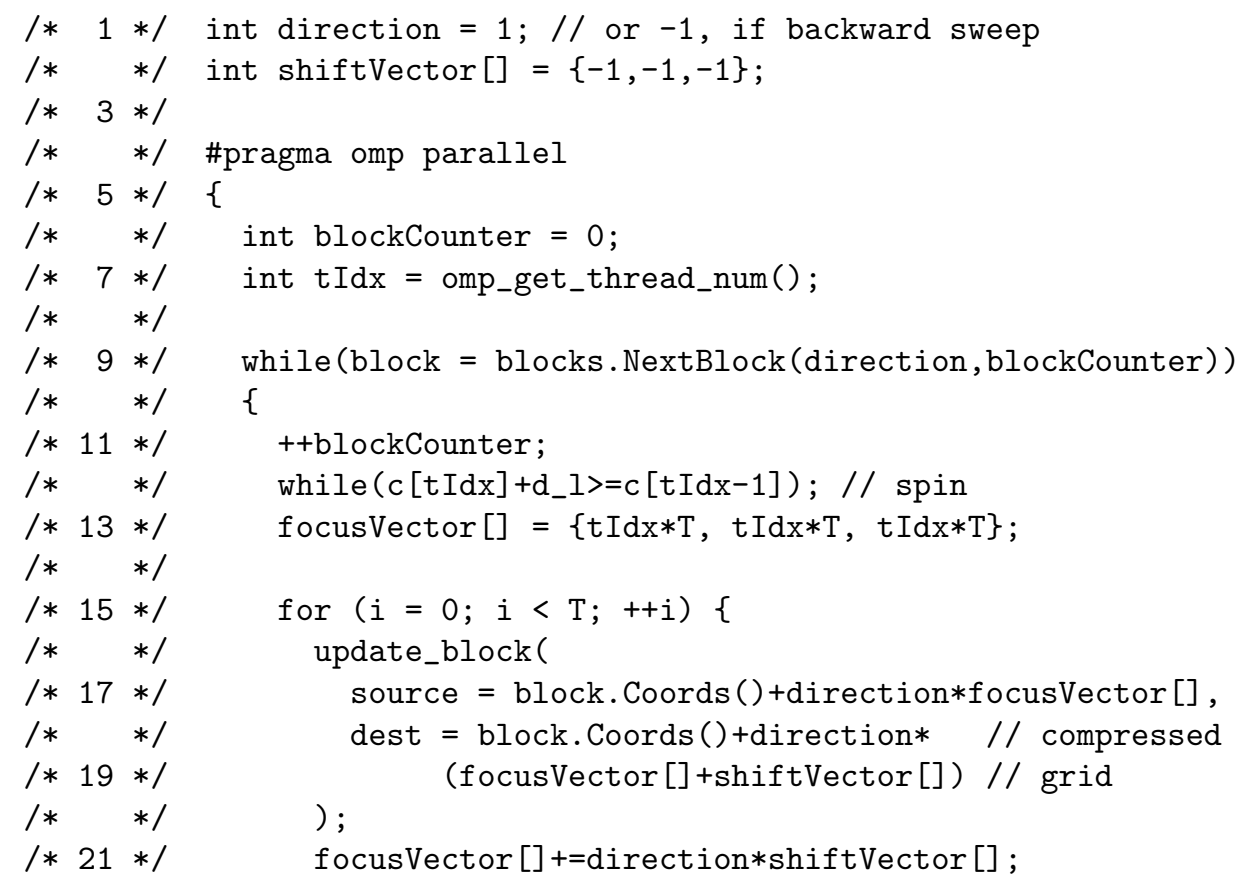




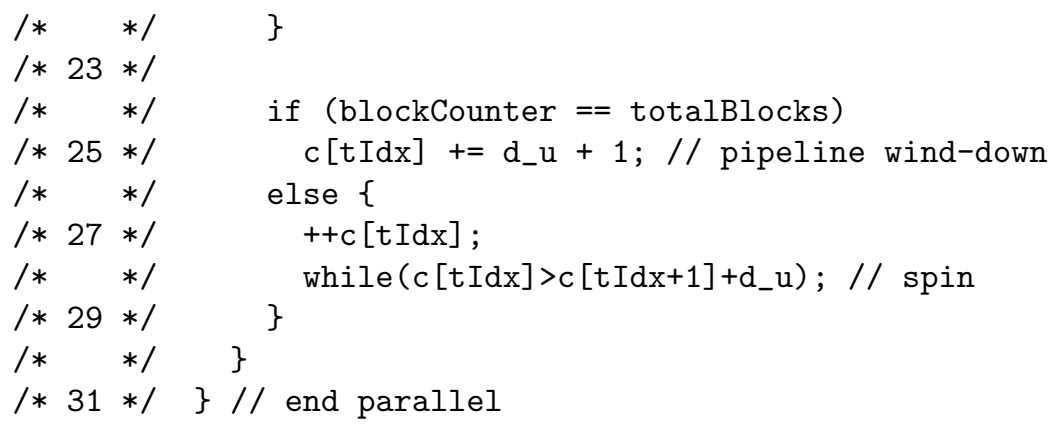

The update_block() function (lines 16-20) performs the actual Jacobi update on a block; the coordinates to use for reading and writing are given in the source and dest parameters, respectively. Lines 12 and 28 contain the spin-waiting loops that implement the correctness and cache size conditions (3), respectively. focusVector [] stores the coordinates of the current block to be updated, and is updated in line 21 to reflect the shift shown in Fig. 1. For a backward sweep, direction is set to -1 ; everything else stays the same.

\subsection{Performance predictions}

In order to get an estimate on the expected performance gain from pipelined temporal blocking, it is essential to identify the bottlenecks that limit scalability on a shared cache. A viable assumption would be that a modified version of (2) is valid even if $t$ updates are performed in cache per stencil (we set $T=1$ ):

$$
P_{0}(t)=\frac{t \cdot M_{\mathrm{um}, 1}}{16 \text { bytes }}[\mathrm{LUP} / \mathrm{s}] \text {. }
$$

Here, $M_{\mathrm{um}, 1}$ is the single-thread bandwidth for a single-stream update benchmark:

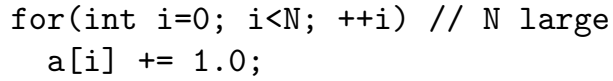

This loop models the data transfer behavior of a compressed grid (and probably temporally blocked) Jacobi solver if we can assume that at least two successive layers of a block fit into a cache level further up in the hierarchy. Then, $t$ updates cause 16 bytes of memory data traffic per stencil. Figure 3 shows performance and scalability for the $T=1$ case within an L3 cache group. The one-socket baseline for the "best" standard Jacobi code is indicated and shows how many cores are required at $T=1$ to match its performance with pipelined temporal blocking. Filled symbols show the prediction based on the bandwidth model (4), using the single-threaded update bandwidth $\left(M_{\mathrm{um}, 1}\right.$ in Table 1). The measurements clearly fall short of this prediction, especially on the Nehalem EP. Our assumption that raw memory bandwidth is always the limiting factor must thus be false. A more careful analysis is in order that takes into account the actual in-cache runtime of the kernel. We shall see that, contrary to popular wisdom, the cache hierarchy and the code execution from L1 cache are not infinitely fast even though the code balance of a particular kernel seems to support this assumption.

The given seven-point Jacobi stencil performs six flops (one multiplication and five additions), six loads (five loads if assuming that variables on the central line can be kept in register) and one store. The Intel Nehalem EP can execute one SIMD multiply, one SIMD add, one 16-byte load and one 16-byte store instruction per cycle. Under 


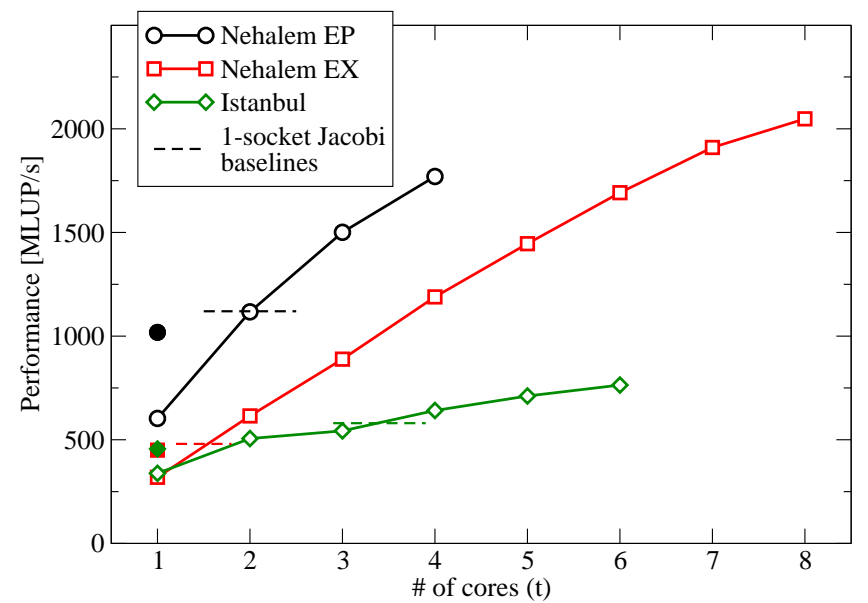

Figure 3: Performance and scalability of the pipelined Jacobi solver, using relaxed synchronization with $T=1$ within an L3 cache group on all three benchmark architectures. Dashed lines indicate the performance of the best standard Jacobi implementation using all threads on a socket. Filled symbols denote the $t=1, T=1$ prediction from a single-threaded update benchmark.
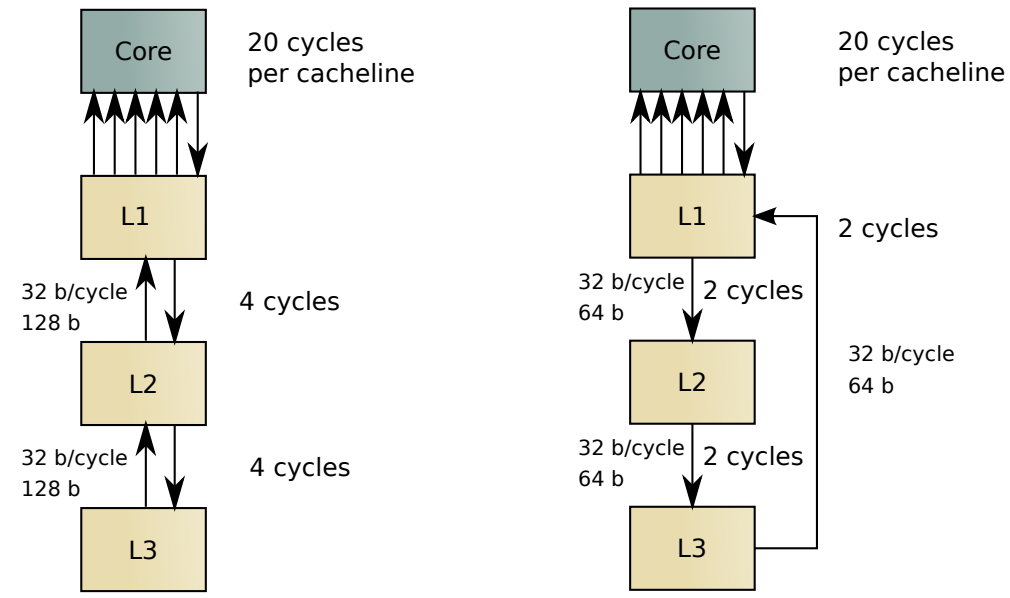

Figure 4: In-cache performance analysis of the compressed grid Jacobi kernel on Intel Nehalem EP (left) and AMD Istanbul (right). Each arrow represents one 64-byte cacheline transfer. The number of cycles required for all transfers between each pair of cache levels is indicated on the right. Those contributions may partially overlap; see text for details. 
the assumption of optimal scheduling and pipelining this results in a minimal runtime of five cycles per vector update ( 20 cycles for a cacheline update) on data in the L1 cache. The performance is limited by the arithmetic instruction throughput as well as by the load/store capabilities. The "lightspeed" performance on our benchmark system is thus $6.4 \mathrm{GF} / \mathrm{s}(1.1 \mathrm{GLUP} / \mathrm{s})$, so the absolute limit for any kind of optimized Jacobi solver is $4.4 \mathrm{GLUP} / \mathrm{s}$ per socket, or a speedup of four compared to the standard Jacobi baseline (see Fig. 3). If the data set does not fit in the L1 cache, additional cacheline transfers are necessary. On Intel processors the caches are inclusive, which means that a valid cacheline is duplicated in all "lower" cache levels. Figure 4 (left) illustrates how the necessary data transfers inside the cache hierarchy contribute to the total runtime if one can assume that at least two successive layers of a block fit into the L1 cache, and blocks are handed down the pipeline through L3. For a detailed introduction to this kind of intra-cache model cf. [12]. The estimated runtime for the L3 cache domain is 28 cycles per cacheline update. Measurements show that the Intel Nehalem processor is able to partially overlap the reloads from L3 to L2 cache with the cycles spent on executing the instructions on data in the L1 cache (however, refills from L2 to L1 can not overlap with L1-register traffic). Hence, the prediction for the duration of one cacheline update (eight successive stencils) in L3 is 24-28 cycles, or, in terms of L3 bandwidth, $B_{\mathrm{j}}=12.2-14.2 \mathrm{~GB} / \mathrm{s}$. Note that the array update benchmark described above runs in 4.7 cycles in L1 (with a theoretical minimum of 4.0) and 12 cycles in L3 per cacheline update. The latter number can be derived from the single-threaded in-L3 bandwidth of the update benchmark shown in Table $11\left(M_{\mathrm{uc}, 1}\right)$. The Nehalem EP and EX CPUs show very similar in-cache behavior for single-threaded execution.

The same scheme can be applied for the AMD Istanbul processor, where our Jacobi stencil is limited by arithmetic instruction throughput (also one SIMD multiply and one SIMD add per cycle). In contrast to the Intel processors, AMD uses an exclusive cache design: L2 and L3 are victim caches and all data loads go directly to the L1 cache. The resulting transfer paths and volumes are illustrated in Fig. 4. The estimated minimum runtime for the compressed grid Jacobi stencil in L3 is 26 cycles, which is equivalent to a bandwidth of $B_{\mathrm{j}}=12.8 \mathrm{~GB} / \mathrm{s}$ on our test machine. Our array update benchmark requires only 18 cycles in L3. Note that the cycle predictions of the in-cache model are lower limits, and even more time is needed per cacheline update in practice. However, they yield a much better prediction than pure bandwidth considerations (L2 and L3 caches often have the same nominal bandwidths as L1).

Hence, the conclusion for the single-threaded case is that the in-cache operations are a major contribution to overall runtime, and cannot be ignored. Since each L1 and L2 cache is assigned to a single core on all architectures in the test bed, the next bottleneck to consider is the shared L3 cache. We assume that it can deliver a maximum aggregate bandwidth to all agents (main memory and cores), and becomes a performance constriction if the sum of all bandwidths hits the maximum. We consider $M_{\mathrm{uc}, \max }$, the aggregate bandwidth for the update benchmark using all cores in the L3 group, to be the upper limit for L3. See Table 1 for performance data.

If $t$ threads are used for pipelined temporal blocking via L3, all of them exert pressure on this cache according to their execution timings as described above. The required memory transfers for bringing a block into cache and for its final eviction add another read and write stream, so that the required bandwidth to reach full scalability is $(t+1) B_{\mathrm{j}}$. For Nehalem EP, if we assume $B_{\mathrm{j}} \approx 10 \mathrm{~GB} / \mathrm{s}$, we expect from the relatively small ratio of $M_{\mathrm{uc}, \max } / B_{\mathrm{j}}$ that pipelined blocking will only just scale up to $t=4$ threads. On the other hand, Nehalem EX should scale well up to eight threads and beyond. Indeed, we see parallel efficiencies around $80 \%$ on both chips up to $t=4$ 


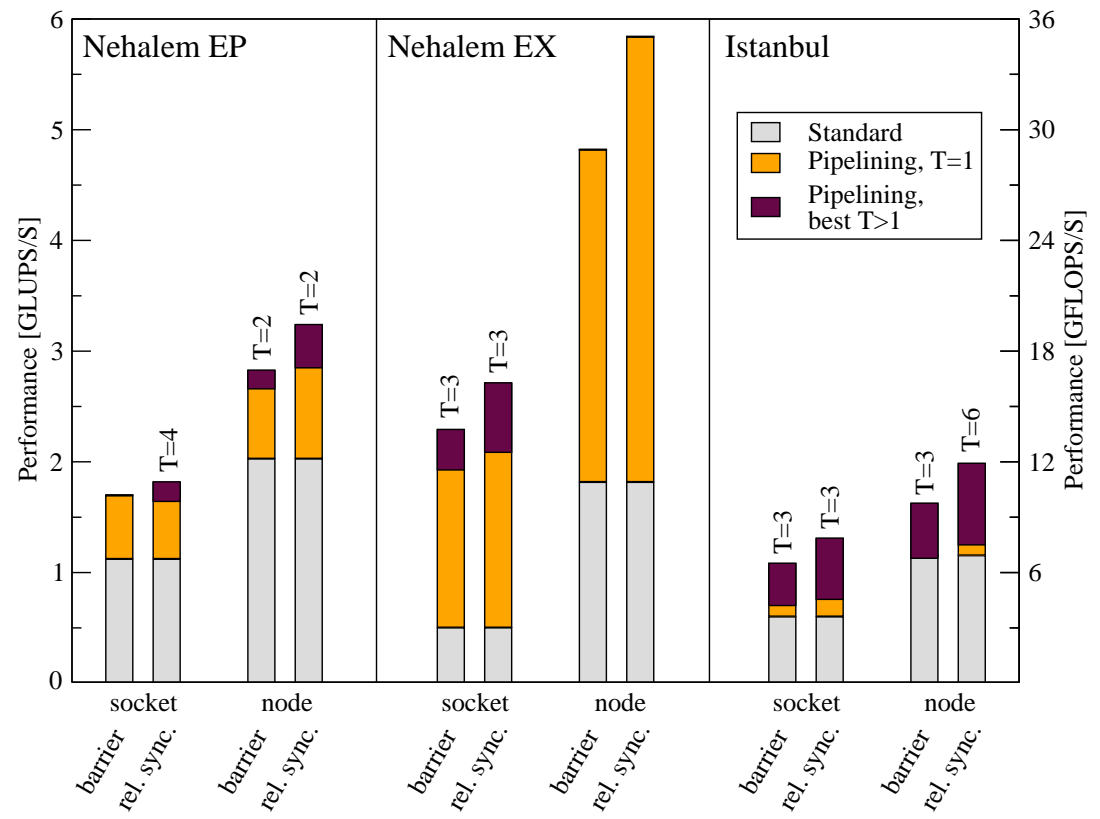

Figure 5: Single-socket and single-node performance results for different variants of pipelined temporal blocking versus the standard version $\left(600^{3}\right.$ grid). $T=1$ and best $T>1$ performance, and the gain from relaxed synchronization are indicated. Optimal values for $T$ were determined empirically.

and $t=8$, respectively (see Fig. 3). In contrast, the AMD Istanbul processor shows mediocre scalability but no typical saturation behavior. As will be shown in the next section, $T=1$ is not an optimal choice here; more updates per block are needed to reach adequate performance. The reason for this is unclear and also unexpected from the low-level performance data we have collected.

\subsection{Socket and node results}

We must stress that the parameter space for temporal blocking schemes, and especially for pipelined blocking, is huge. The optimal choices reported here have been obtained experimentally by parameter sweeps, with some guidance from experience with older codes.

Figure 5 shows single-socket (one team) and node results (two teams) on our test systems for a fixed problem size of $600^{3}$ and different algorithmic variants: the standard Jacobi solver baseline, pipelined temporal blocking with $T=1$ and $t$ being equal to the number of cores in a socket (or system), and pipelined temporal blocking with $T$ chosen optimally. For all versions, the difference between a global barrier and our relaxed synchronization scheme is also indicated. The standard Jacobi data was obtained with a blocksize of roughly $600 \times 20 \times 20\left(b_{x} \times b_{y} \times b_{z}\right)$; it is well known that due to the hardware prefetching mechanisms on current $x 86$ designs, a long inner loop (comparable to the page size) is favorable [2]. While the results are rather insensitive to the blocksizes in $y$ and $z$ directions as long as the cache size restrictions are observed, the inner loop length is also decisive for good performance on the temporally blocked 


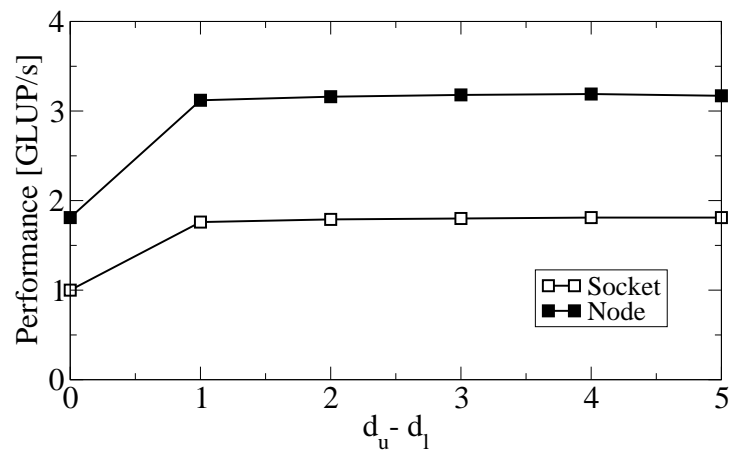

Figure 6: Influence of pipeline looseness on the socket (open symbols) and node level (filled symbols). The case $d_{\mathrm{u}}-d_{1}=0$ represents a rigid "lockstep", which is obviously hazardous. Data was taken on Nehalem EP, but the general characteristic is very similar on all architectures.

versions, and depends on $T$. Best performance is achieved around $b_{x} \approx 120$ if $T>1$, in contrast to the $T=1$ case where $b_{x}$ should be chosen as large as possible.

The possible gain from pipelined temporal blocking varies strongly across the benchmark architectures. As expected, a large speedup is achieved if the gap between a socket's memory bandwidth and its L3 saturation bandwidth is large. Nehalem EP is exceptional in the sense that this discrepancy is relatively small; also, using more cores with the same L3 design (as implemented in the Intel "Westmere" chip) would not make sense at all. This makes temporal blocking schemes of any kind less beneficial than on other, more bandwidth-starved architectures. With a speedup of 5-6 on the socket and of 3 on the full node, Nehalem EX clearly dominates due to its scalable L3 cache design, large number of cores, and (in our EA system) low memory bandwidth. Both Intel processors get most of the speedup already at $T=1$, whereas the AMD Istanbul requires more time steps per block. A possible reason could be inefficient hardware prefetching within the cache hierarchy [12]; latencies have less impact if more work is done before fetching the next block.

The benefit of relaxed synchronization naturally goes up with the number of cores and sockets in the team, and gets as large as $15 \%$ on Nehalem EX. Hence, we expect efficient synchronization schemes to become more and more important in the future as the core count per socket increases. It is certainly possible to achieve even larger gains by choosing a smaller (and then non-optimal) block size.

The optimal range of values for $d_{\mathrm{u}}$, the upper limit for the distance of neighboring threads, was determined to be $2-4$ with the block sizes chosen, largely independent of architecture (see Fig. 6). This allows for sufficient looseness in the pipeline without running the danger of blocks falling out of cache before the team's rear thread has done its updates on them. Compared to the "lockstep" case $d_{\mathrm{l}}=d_{\mathrm{u}}=1$, a performance gain of about $80 \%$ can be observed for Nehalem EP. Of course, $d_{\mathrm{u}}$ and the blocksize are strongly coupled, and larger blocks would require smaller $d_{\mathrm{u}}$, but we could not find better combinations than the ones reported here. A finite team delay $d_{\mathrm{t}}$ only has a very slight impact on this architecture (about 3\% improvement for $d_{\mathrm{t}}=8$ ), and its influence will not be studied further.

Scalability of the pipelined code is not perfect across sockets, since proper NUMA placement cannot be enforced, as described above. However, distributed-memory par- 


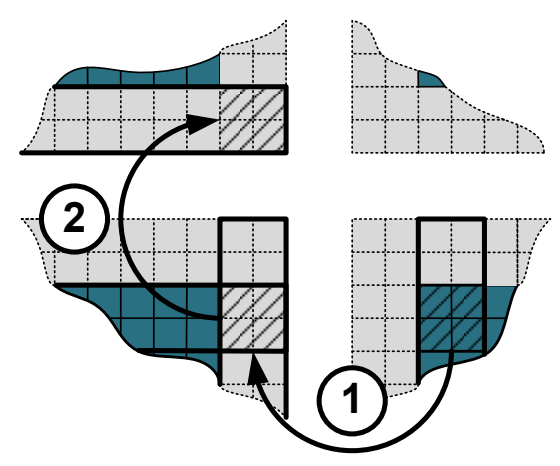

Figure 7: Multi-layer halo communication. Each halo is transmitted consecutively along the three coordinate directions, avoiding direct communication across edges and corners [13].

allelization (see next section) can employ one process per socket, eliminating the need for first-touch parallel placement.

\section{Distributed-memory parallelization}

\subsection{Multi-halo exchange}

The parallel temporal blocking schemes described above work on multicore-based shared-memory architectures. Stencil algorithms are usually straightforward to parallelize on distributed-memory systems using domain decomposition and halo layer exchange, and the temporally blocked Jacobi code is no exception: The computational domain is decomposed as usual, but instead of a single halo, $h$ layers must be exchanged after $h=n \cdot t \cdot T$ updates have been performed per subdomain. Subdomains overlap by $h-1$ grid layers, and extra work is involved on the boundaries because update number $s$ covers a domain that is $h-s$ layers larger in each direction. The amount of data communication per stencil update is roughly the same as for no temporal blocking, except for edge and corner contributions, which only become important on very small subdomains (see below). An important side effect of multi-layer halo exchange is that communication takes place across subdomain edges and corners. Latency-dominated small messages can be avoided by transmitting halos consecutively along the three coordinate directions [13] (see Fig. 7).

The question arises whether the use of multi-layer halo exchange has any significant impact on performance. Two factors lead to opposite effects here: If the subdomain surface area is very small, aggregating multiple messages into one may be beneficial; effective bandwidth rises dramatically with growing message size in the latencydominated regime. On the other hand, the surface to volume ratio is large in this limit, leading to a significant overhead from communication and extra halo work. The different contributions to execution time ("bulk" and additional "face" stencil updates, and halo exchange) can be calculated, assuming a simple latency/bandwidth model for network communication and no overlap between calculation and data transfer.

While only simple algebra is involved, the resulting expressions are very complex, so we restrict ourselves to a graphical analysis. The main panel of Fig. 8 shows the pre- 


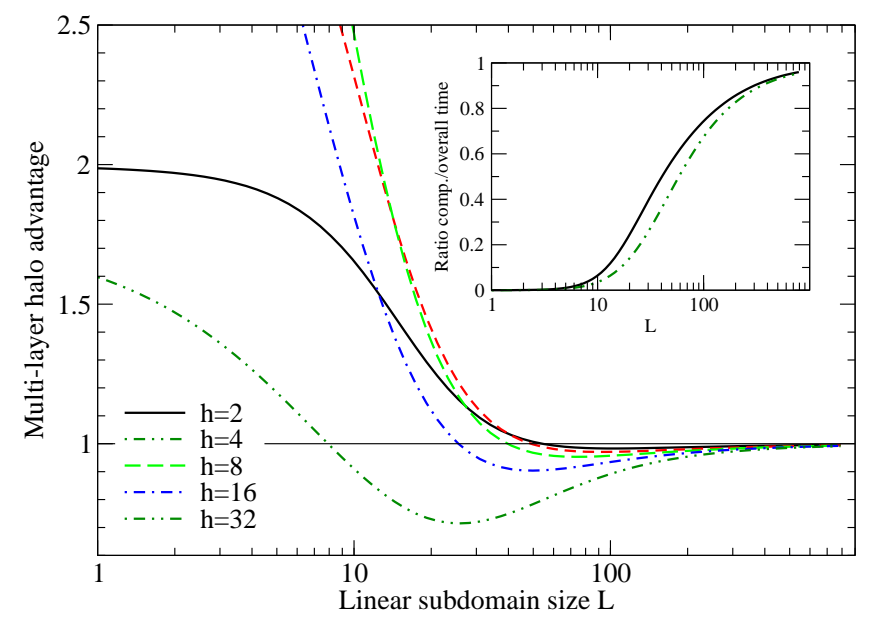

Figure 8: Theoretical multi-layer halo advantage versus linear subdomain size $L$ for different halo widths $h$. Parameters are set for a vector-mode hybrid Jacobi solver on a QDR-IB network and a per-node performance of $2000 \mathrm{MLUP} / \mathrm{s}$ (see text for details). Inset: Ratio of computation versus overall time ("computational efficiency") for the corner cases $h=2$ and $h=32$.

dicted ratio of execution times between a standard one-layer halo version and $h$-layer exchange for cubic subdomains of size $L^{3}$ and different $h$. We have set the parameters for a QDR-InfiniBand network here, with an asymptotic (large-message) unidirectional bandwidth of $3.2 \mathrm{~GB} / \mathrm{s}$ and a latency of $1.8 \mu \mathrm{s}$. The single-node performance was assumed to be $2000 \mathrm{MLUP} / \mathrm{s}$, independent of $L$ (which only roughly holds in practice). As expected, multi-layer halos have no influence at large subdomain sizes. As the domain gets smaller $(20 \lesssim L \lesssim 100)$, extra halo work starts to degrade performance, but a relevant impact can only be expected at $h \gtrsim 16$. At even smaller $L \lesssim 20$, the positive effect of message aggregation over-compensates the halo overhead, leading to substantial performance gains. Although this looks like a good result, the ratio of computation time versus overall execution time as shown in the inset of Fig. 8 proves that the algorithm is strongly communication-limited below $L \approx 100$, such that parallel efficiency is very low. Any gain obtained by sophisticated temporal blocking is squandered by communication overhead in this limit.

Note that this simple model disregards some important effects like switching of message protocols, overhead for copying to and from message buffers, load imbalance, etc. Its purpose is to get a rough idea about where to expect benefits from distributedmemory parallelization of pipelined blocking with multi-layer halos.

\subsection{Distributed-memory results}

Implementation of the hybrid MPI/OpenMP pipelined Jacobi code was straightforward, with no explicit or implicit overlapping of communication and computation. The MPI library used (Intel MPI 3.2.2.006) does not support asynchronous non-blocking transfers. Profiling has shown that copying halo data from boundary cells to and from intermediate message buffers causes about the same overhead as the actual data transfer over the QDR-InfinBand interconnect. We have investigated the performance of our distributed-memory multi-halo pipelined Jacobi solver in strong and weak scaling 


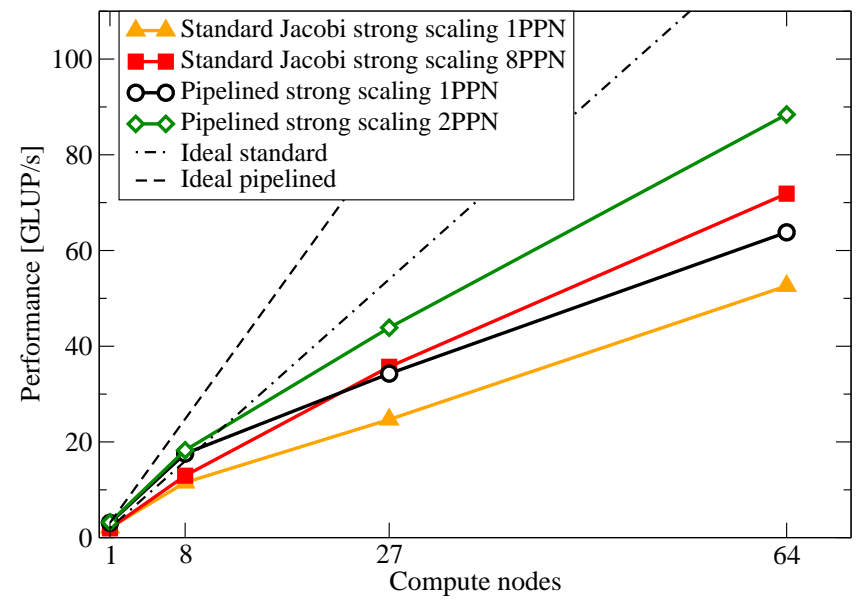

Figure 9: Distributed-memory parallel performance (strong scaling) of the standard and the multi-halo pipelined Jacobi solvers with relaxed synchronization, at a problem size of $600^{3}$.

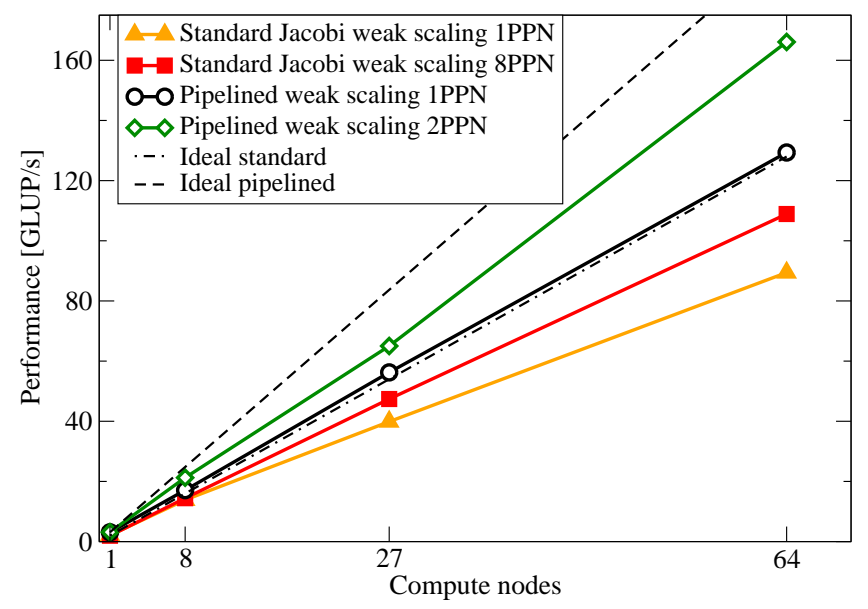

Figure 10: Distributed-memory parallel performance (weak scaling) of the standard and the multi-halo pipelined Jacobi solvers with relaxed synchronization, at a problem size of $600^{3}$ per process. 
scenarios, respectively, and compared to the standard solver.

Figure 9 shows performance data for strong scaling at a constant overall problem size of $600^{3}$ on 1 to 64 nodes. The standard Jacobi code (triangles and squares) was run with one MPI process per core (8PPN) and in "hybrid vector" mode (1PPN), the latter being clearly inferior. The "2PPN" multi-halo pipelined version, which uses one MPI process per socket, outperforms the "1PPN" version considerably because of improved NUMA placement. However, it is just a slight progress compared to the standard Jacobi code, and most of the performance gain achieved by pipelined blocking on the node level is lost due to the dominating communication overhead. This is exactly in line with the results from the multi-halo performance model in the previous section.

The situation changes with weak scaling. Figure 10 shows weak scaling data at a problem size of $600^{3}$ per node. Again, although communication is now less important, the hybrid vector version of the standard solver performs worse than the pure MPI version. The multi-halo code with one MPI process per socket, however (2PPN), can now maintain about $80 \%$ of the single-node speedup also in the distributed-memory parallel case. Note that this results depends on the problem size and the communication network; on a slow network, or with a much smaller problem size per node, communication may still play a dominant role and cancel out the gain from temporal blocking.

\section{Summary and outlook}

We have demonstrated that multicore-aware pipelined temporal blocking can lead to a substantial performance improvement for the Jacobi algorithm on current multicore architectures (Intel Nehalem EP/EX, AMD Istanbul). Substitution of a global barrier by relaxed synchronization between neighboring threads adds to the benefits. A simple bandwidth-based performance model was shown to be insufficient to predict the expected speedup at low thread counts. Instead, a cycle-by-cycle analysis of inter-cache traffic for the Jacobi algorithm revealed that instruction execution and cache bandwidth rather than memory bandwidth are the limiting factors in those cases.

In comparison to earlier, more bandwidth-starved processors, the potential gain on Nehalem EP is limited due to its high memory bandwidth and relatively slow L3 cache. However, future multicore processors can be expected to be less balanced, and thus profit more from temporal blocking, especially if the shared outer-level cache is scalable. Our Nehalem EX test system could serve as a "blueprint" of such a design; its low memory bandwidth and very fast L3 cache make temporal blocking extremely beneficial. The performance characteristics of the AMD Istanbul processor, although it is on par with Nehalem EP by raw numbers, are poorly understood and worth investigating further.

We have also shown, theoretically and in practice, under which circumstances it is possible to port the temporal blocking speedup to a distributed-memory parallel (hybrid) code. A hybrid, temporally blocked lattice Boltzmann flow solver based on the principles presented in this work is under development.

Further optimizations are possible: One main drawback of our method is that all cores in a node form a single large pipeline, inhibiting optimal ccNUMA placement. This could be corrected by a domain decomposition strategy similar to the one demonstrated in Ref. [4], but experience shows that in practice (e.g., if a pipelined stencil code should be used as a smoother component in a Multigrid algorithm) it is usually best to use one MPI process per ccNUMA domain anyway. 


\section{Acknowledgment}

Lively discussions with Darren Kerbyson, Thomas Zeiser, and Johannes Habich are gratefully acknowledged. We are indebted to Intel for providing early access hardware and technical information. This work was supported by BMBF under grant No. 01IH08003A (project SKALB), and by the Competence Network for Scientific High Performance Computing in Bavaria (KONWIHR) through the project OMPI4PAPPS.

\section{References}

[1] B. Bergen, F. Hülsemann, U. Rüde: Is $1.7 \times 10^{10}$ Unknowns the Largest Finite Element System that Can Be Solved Today? In: ACM/IEEE (Ed.): Proceedings of the ACM/IEEE SC 2005 Conference (Supercomputing Conference '05, Seattle, Nov 12-18, 2005).

[2] K. Datta et al.: Stencil Computation Optimization and Auto-tuning on State-of-the-art Multicore Architectures. Proc. SC2008. DOI:10.1145/1413370.1413375

[3] K. Datta et al.: Optimization and performance modeling of stencil computations on modern microprocessors. SIAM Review 51(1), (2009) 129-159. DOI:10.1137/070693199

[4] G. Wellein, G. Hager, T. Zeiser, M. Wittmann and H. Fehske: Efficient temporal blocking for stencil computations by multicore-aware wavefront parallelization. Proc. COMPSAC 2009. DOI:10.1109/COMPSAC. 2009.82

[5] J. Treibig, G. Hager: Introducing a Performance Model for Bandwidth Limited Loop Kernels. Workshop on Memory Issues on Multi- and Manycore Platforms, Proc. PPAM 2009. http://arxiv.org/abs/0905.0792

[6] D. Wonnacott: Using Time Skewing to Eliminate Idle Time due to Memory Bandwidth and Network Limitations. Proc. IPDPS 2000. DOI:10.1109/IPDPS . 2000.845979

[7] G. Jin, J. Mellor-Crummey, and R. Fowler: Increasing temporal locality with skewing and recursive blocking. Proc. SC2001. DOI:10.1145/582034.582077

[8] M. Kowarschik: Data Locality Optimizations for Iterative Numerical Algorithms and Cellular Automata on Hierarchical Memory Architectures. Dissertation, SCS Publishing House, Erlangen (2004). http://www10. informatik.uni-erlangen.de/ Publications/Dissertations/Diss_Kowarschik_2004.pdf

[9] M. Frigo and V. Strumpen: Cache oblivious stencil computations. Proc. ICS 2005. DOI:10.1145/1088149.1088197

[10] S. Kamil, K. Datta, S. Williams, L. Oliker, J. Shalf, and K. Yelick: Implicit and explicit optimizations for stencil computations. Proc. MSPC 2006. DOI:10.1145/1178597.1178605

[11] T. Zeiser, G. Wellein, A. Nitsure, K. Iglberger, U. Rüde, G. Hager: Introducing a parallel cache oblivious blocking approach for the lattice Boltzmann method. Progress in CFD, vol. 8, No. 1-4, pp. 179-188, 2008.

[12] J. Treibig, G. Hager and G. Wellein: Multi-core architectures: Complexities of performance prediction and the impact of cache topology. Under review. http://arxiv . org/abs/0910.4865

[13] C. Ding and Y. He: A ghost cell expansion method for reducing communications in solving PDE problems. Proc. SC2001. DOI:10.1145/582034. 582084

[14] M. Wittmann: Potentials of temporal blocking for stencil-based computations on multicore systems. Master's Thesis, University of Applied Sciences Nuremberg, March 2009. http://www.hpc.rrze.uni-erlangen.de/Projekte/stencil.shtml 